\title{
Group oriented interpersonal therapy reduced postpartum depression in women at risk
}

Zlotnick C, Johnson SL, Miller IW, et al. Postpartum depression in women receiving public assistance: pilot study of an interpersonal-therapy-oriented group intervention. Am J Psychiatry 2001 Apr;158:638-40.

\section{QUESTION: Does a weekly 4 session group intervention based on the principles of interpersonal psychotherapy given to at risk pregnant women reduce the occurrence of postpartum major depression?}

Design

Randomised (unclear allocation concealment*), unblinded*, controlled trial with follow up to 3 months after giving birth.

Setting

Prenatal clinic at a general hospital in Providence, Rhode Island, USA.

\section{Patients}

37 pregnant women receiving public assistance who were at 20-32 weeks gestation and had $\geqslant 1$ risk factor for postpartum depression (ie, a previous episode of depression or postpartum depression, mild to moderate levels of depressive symptoms, poor social support, or a life stressor within the previous 6 mo). 35 women (mean age 23 y) completed the study.

\section{Intervention}

17 women were assigned to the intervention group, Survival Skills for New Moms, involving 4 sixty minute group sessions over a 4 week period. The sessions provided education, focused on depression, role transitions and setting goals, and taught skills for resolving interpersonal conflicts and developing supports. 18 women were assigned to treatment as usual.

\section{Main outcome measures}

Scores on the Beck Depression Inventory (BDI), reliable improvement after the intervention defined as those whose level of functioning moved within the range of the functional population reflected by a reliable change index $>1.96$ for the BDI, and number of women who met criteria for major depression.

Source of funding:

Klingenstein Third

Generation

Foundation.

For correspondence: Dr C Zlotnick, Butler Hospital, 345

Blackstone Boulevard,

Providence, RI 02906,

USA. Fax +1401455

6293.

\section{Main results}

Change in the BDI scores from before to after the intervention was greater in the intervention group and in the right direction than in the control group (from 13.0 to $8.4 v 9.2$ to $11.3, \mathrm{p}=0.001$ ). The number of women who had a reliable improvement after the intervention did not differ between the 2 groups but no women in the intervention group developed postpartum depression compared with $6(33 \%)$ in the control group (table).

Group oriented interpersonal therapy (IT) v treatment as usual (control) at 3 months postpartum in women at riskt

\begin{tabular}{lllll} 
Outcomes & IT & Control & RBI (95\% Cl) & NNT (Cl) \\
\hline Reliable improvement & $35 \%$ & $11 \%$ & $218 \%(-140$ to 1167$)$ & Not significant \\
\hline & IT & Control & RRR (Cl) & NNT (CI) \\
\hline Postpartum depression & $0 \%$ & $33 \%$ & $100 \%(41$ to 100$)$ & $3(2$ to 9$)$ \\
\hline
\end{tabular}

\section{Conclusion}

A 4 session interpersonal therapy oriented group intervention reduced the occurrence of postpartum depression in a group of financially disadvantaged women.

*See glossary.

\section{COMMENTARY}

Postpartum depression is common among single, ${ }^{1}$ economically disadvantaged women who have small social networks, a family history of depression or a previous personal history of depression, concurrent substance abuse, and a history of trauma. It is important not only as a highly prevalent, distressing, and costly condition but, even more importantly, because it affects the health and development of the newborn and of other young children in the family. ${ }^{2}$

Zlotnick et al show how a relatively non-intensive intervention reduced the relative incidence of depression over the 3 months after childbirth. The sessions, as described, dealt with identifying the symptoms of depression, conflict resolution, goal attainment, and social networking. The patients in this study were poor; a majority were single mothers. They do not appear to have been faced with the choice of discontinuing or initiating antidepressants-frequently a very problematic decision for mothers and for physicians. There is no mention of important coexisting factors such as breast feeding, substance abuse, insomnia, number of children, violence, or family history of mood disorder. This study needs to be replicated using a substantial cohort of women from various socioeconomic and educational backgrounds, co-varying out the large number of known contributory factors to the development of postpartum depression and, ideally, using a depression scale developed specifically for this population, such as the Edinburgh Postnatal Depression Scale. The BDI, used in this study, includes somatic symptoms (fatigue, insomnia) that are universal after childbirth and not necessarily indicative of depression.

Nevertheless, even this small study alerts primary care physicians to the need of providing educational and motivational resources to pregnant women, alerting them to the important risk factors for postpartum depression, and equipping new mothers with ways of averting or solving the problems they are about to face. \section{Centre for Addiction and Mental Health Toronto, Ontario, Canada}

Mary V Seeman, MD

1 O'Hara MW, Swain AM. Rates and risk of postpartum depression-a meta-analysis. Int Rev Psychiatry 1996;8:3754 .

2 O'Hara MW. Postpartum depression: causes and consequences. New York: Springer-Verlag, 1995. 